\title{
Article \\ Effect of COVID-19 on Thoracic Oncology Surgery in Spain: A Spanish Thoracic Surgery Society (SECT) Survey
}

\author{
Néstor J. Martínez-Hernández ${ }^{1, *}$, Usue Caballero Silva ${ }^{2}$, Alberto Cabañero Sánchez ${ }^{2}$, \\ José Luis Campo-Cañaveral de la Cruz ${ }^{3}$, Andrés Obeso Carillo ${ }^{4} \mathbb{D}$, José Ramón Jarabo Sarceda 5 , \\ Sebastián Sevilla López ${ }^{6}$, Ángel Cilleruelo Ramos ${ }^{7}{ }^{\circledR}$, José Luis Recuero Díaz ${ }^{8}$, Sergi Call ${ }^{9}$, \\ Felipe Couñago ${ }^{10,11,12}$, Florentino Hernando Trancho ${ }^{5}$ \\ and on behalf of the Scientific Committee of the Spanish Thoracic Surgery Society
}

check for

updates

Citation: Martínez-Hernández, N.J.;

Caballero Silva, U.;

Cabañero Sánchez, A.;

Campo-Cañaveral de la Cruz, J.L.;

Obeso Carillo, A.; Jarabo Sarceda, J.R.;

Sevilla López, S.; Cilleruelo Ramos, Á.;

Recuero Díaz, J.L.; Call, S.; et al. Effect of COVID-19 on Thoracic Oncology Surgery in Spain: A Spanish Thoracic Surgery Society (SECT) Survey. Cancers 2021, 13, 2897. https:/ / doi.org/10.3390/cancers13122897

Academic Editor: Tetsuya Mitsudomi

Received: 30 April 2021

Accepted: 2 June 2021

Published: 9 June 2021

Publisher's Note: MDPI stays neutral with regard to jurisdictional claims in published maps and institutional affiliations.

Copyright: (c) 2021 by the authors. Licensee MDPI, Basel, Switzerland. This article is an open access article distributed under the terms and conditions of the Creative Commons Attribution (CC BY) license (https:/ / creativecommons.org/licenses/by/ $4.0 /)$.
1 Thoracic Surgery, Hospital Universitari de la Ribera, 46600 Alzira, Spain

2 Thoracic Surgery, Hospital Universitario Ramón y Cajal, 28034 Madrid, Spain; usue.caballero@salud.madrid.org (U.C.S.); Alberto.cabanero@salud.madrid.org (A.C.S.)

3 Thoracic Surgery, Hospital Universitario Puerta de Hierro-Majadahonda, 28222 Majadahonda, Spain; campo-canaveral.delacruz@salud.madrid.org

4 Thoracic Surgery, Hospital Clínico Universitario de Santiago, 15706 Santiago de Compostela, Spain; gerardo.andres.obeso.carillo@sergas.es

5 Thoracic Surgery, Hospital Clínico San Carlos, 28040 Madrid, Spain; joseramon.jarabo@salud.madrid.org (J.R.J.S.); florentino.hernando@salud.madrid.org (F.H.T.)

6 Thoracic Surgery, Hospitalario Universitario Ciudad de Jaén, 23007 Jaén, Spain; sebastian.sevilla.sspa@juntadeandalucia.es

7 Thoracic Surgery, Hospital Clínico de Valladolid, 47003 Valladolid, Spain; acilleruelo@saludcastillayleon.es

8 Thoracic Surgery, Instituto de Investigación Sanitaria de Aragón, Hospital Universitario Miguel Servet, 50009 Zaragoza, Spain; jlrecuero@salud.aragon.es

9 Thoracic Surgery, Hospital Universitari Mútua Terrassa, 08221 Terrassa, Spain; scall@mutuaterrassa.cat

10 Radiation Oncology, Hospital Universitario Quirónsalud, 28223 Pozuelo de Alarcón, Spain; felipe.counago@quironsalud.es

11 Radiation Oncology, Hospital La Luz, 28003 Madrid, Spain

12 Radiation Oncology, Universidad Europea de Madrid, 28670 Villaviciosa de Odón, Spain

* Correspondence: martinez_nes@gva.es or nessmartinez@gmail.com; Tel.: +34-717122000

Simple Summary: After the first wave of COVID-19, the Spanish Society of Thoracic Surgeons (SECT) surveyed its members to assess the impact of the pandemic on thoracic oncology surgery in one of the counties most affected by the virus. In May 2020, all SECT members were invited to complete a 40 -item, multiple choice questionnaire by e-mail. The response rate was $19.0 \%$. Surgical activity decreased by $95.7 \%$, with $41.5 \%$ of centers performing surgery only in oncologic cases and $11.7 \%$ only in emergencies. More than half (56\%) of multidisciplinary tumour board meetings (56\%) were cancelled or conducted online. Standard protocols for early-stage disease were modified in $62.9 \%$ of centers. The results of this survey show that the COVID-19 pandemic severely limited thoracic oncology surgery activity. Here we describe and discuss the impact of the pandemic on thoracic surgery in Spain.

Abstract: After the first wave of COVID-19, the Spanish Society of Thoracic Surgeons (SECT) surveyed its members to assess the impact of the pandemic on thoracic oncology surgery in Spain. In May 2020, all SECT members were invited to complete an online, 40-item, multiple choice questionnaire. The questionnaire was developed by the SECT Scientific Committee and sent via email. The overall response rate was $19.2 \%$. The respondents answered at least $91.5 \%$ of the items, with only one exception (a question about residents). Most respondents (89.3\%) worked in public hospitals. The reported impact of the pandemic on routine clinical activity was considered extreme or severe by $75.5 \%$ of respondents ( $25.5 \%$ and $50 \%$, respectively). Multidisciplinary tumour boards were held either with fewer members attending or through electronic platforms ( $44.6 \%$ and $35.9 \%$, respectively). Surgical activity decreased by $95.7 \%$, with $41.5 \%$ of centers performing surgery only on oncological patients and $11.7 \%$ only in emergencies. Nearly $60 \%$ of respondents reported modifying standard protocols for early-stage cancer and in the preoperative workup. Most centers $(\approx 80 \%)$ reported using 
full personal protective equipment when operating on COVID-19 positive patients. The COVID-19 pandemic severely affected thoracic oncology surgery in Spain. The lack of common protocols led to a variable care delivery to lung cancer patients.

Keywords: lung cancer; surgical treatment; COVID-19

\section{Introduction}

Lung cancer is the most lethal type of cancer, accounting for 1.76 million deaths annually [1]. The treatment of lung cancer is multimodal and multiple strategies are available depending on the stage at diagnosis. Approximately $25-30 \%$ of patients are diagnosed with early-stage disease [2]. In many of these patients, lung resection is the treatment of choice [3]. Although lung cancer is often considered an epidemic, the emergence of a new coronavirus variant in December 2019-SARS-CoV-2, which causes the disease known as COVID-19-quickly eclipsed lung cancer and most other health conditions. A few months later, on 11 March 2020, the World Health Organization officially declared a pandemic $[4,5]$. In only one year, COVID-19 has directly or indirectly caused over 3 million deaths worldwide [6], forcing governments around the world to implement strict measures restricting the free movement of citizens and bringing the economy to a halt [7-9].

The last major pandemic was the flu of 1918 [10] when the field of medicine and hospital organisation were very different from the present. Moreover, at that time, the limited therapeutic arsenal for lung cancer did not yet include surgery [11], which explains the unprecedented impact of the current pandemic on the diagnosis and treatment of lung cancer.

In Spain, the epidemiological situation worsened quickly after the initial outbreak of the virus, reaching nearly catastrophic proportions. In the first wave, Spain had the highest excess mortality rate per 100,000 inhabitants in Europe and also the highest relative excess number of deaths, which was only surpassed among males in certain regions of the United Kingdom [12]. Strict measures were imposed to restrict the movement of the population during the early months of the pandemic from March to May, 2020. Nevertheless, COVID-19 patients accounted for more than $100 \%$ of hospital occupancy $(105 \%)$ in some regions, which required the addition of more beds in cafeterias, libraries, gyms, etc. In some cases, intensive care unit (ICU) occupancy rates were as high as 300\% [13], and field hospitals were created in several cities to accommodate patient overflow [14]. Hospitals were overwhelmed during this first wave, which had a major impact on the treatment of all non-COVID-19 conditions. In many cases-and lung cancer was no exception-surgery had to be cancelled or postponed indefinitely [15].

In this context, in which operating room availability was greatly reduced or even completely unavailable due to closures, the main national and international scientific societies issued recommendations for the management of patients with thoracic cancer. Those recommendations called for triaging patients for surgery based on the theoretical deferability of the operation, taking into account the safety of patients and surgical teams alike, and adapting the recommendations to the specific conditions in each region or country [16,17]. These guidelines, together with the use of COVID-19-free areas of the hospital, were particularly important in Spain, in which the high incidence of SARS-CoV-2 and consequent hospital overload had a major impact on surgical procedures. Importantly, this strategy allowed for the surgical treatment of selected patients with little to no excess morbidity and mortality $[18,19]$.

To evaluate the true impact of the pandemic on thoracic surgery departments in a country powerfully affected by the first wave of the COVID-19 pandemic, the Spanish Society of Thoracic Surgery (SECT) carried out an anonymous survey to obtain first-hand information from its members. The main aim of the present study is to report and discuss the results of that survey to provide insight into the treatment of lung cancer during 
one of the most adverse scenarios imaginable. The survey had three main objectives regarding the surgical treatment of lung cancer in Spain during the first wave of COVID-19: (1) to audit the activity of the multidisciplinary tumor board (MTB) and decision-making during this challenging period; (2) to determine the extent to which the pandemic affected the preoperative diagnostic pathway; and (3) to determine the impact on the surgical procedures and protocols.

\section{Materials and Methods}

Members of the SECT, the association that represents all thoracic surgeons in Spain, were invited by email to participate in this survey. The first invitation was sent on 7 May 2020 to the 471 members of the SECT. The survey was created on the SurveyMonkey platform (www.surveymonkey.co.uk, accessed on 5 May 2020). Reminders were subsequently sent by email on 12 May and 20 May. The survey remained open until 29 May 2020.

The survey was designed by the SECT Scientific Committee and consisted of 40 multiple choice questions. Of these, nine questions were designed to assess the members sociodemographic background and the general operation of their hospital. There were 28 questions on the impact of the pandemic on the surgical treatment of thoracic cancer, two on the quality of the scientific studies on COVID-19 published to date, and one on the impact of the pandemic on training of thoracic surgeons. The 28 questions about the impact on surgery focused on the following areas: functioning of the MTB and waiting lists (five questions); preoperative studies (four questions); aspects related to the surgical procedure, postoperative care, and the management of pleural fluids (10 questions); SARS-CoV-2 diagnosis and testing in patients scheduled for surgery (five questions); and protective measures for physicians (four questions). The estimated time to complete the survey was nine minutes. The complete survey is shown in Table 1.

Table 1. Survey: questions and options.

Sociodemographics and Hospital-Related Data

1. What country do you work in?

- Spain

- Other

2. What type of institution do you work at?

- University Public hospital

- Non-University Public hospital

- Private hospital

- University Private hospital

3. How many beds does your hospital have?

- 100 beds or less

- $\quad$ Between 100 and 400 beds

- $\quad$ Between 400 and 800 beds

- $\quad>800$ beds

4. Did your hospital have to increase the number of beds?

- No

- Yes, only for ICU beds

- Yes, it was necessary to increase hospital beds

- Yes, it was necessary to use other installations (gym, other centers, ... )

5. Was it necessary to adapt other spaces in the hospital to treat COVID-19 patients (e.g., gymnasium, library, cafeteria, field hospital, etc.)?

- Yes

- $\mathrm{No}$ 
Table 1. Cont.

\section{Sociodemographics and Hospital-Related Data}

6. Did you refer patients from your center to hotels or other facilities?

- Yes

- No

7. How affected was the normal functioning of your hospital?

- Not at all

- $\quad$ Slightly (some minor elective surgeries has been posponed, not other changes)

- Moderately (Significant emergency department activity, more surgeries postponed, less ICU beds available for non-COVID-19 patients)

- Severe (significant emergency department activity, only medically or oncologically urgent surgeries are executed, minority of ICU beds available for non COVID-19 patients)

- $\quad$ Extreme (hospital care is insufficient for this pandemic: shortness of beds, staff, supplies and ICU resources)

8. Have healthcare staff been tested for SARS-CoV-2?

- No

- Yes, everyone

- Yes, but only after having symptoms

- $\quad$ Yes, only after exposure to COVID-19 patient

- Yes, after having symptoms or exposure to COVID-19 patient

- The criteria is not well defined

9. Has any member of the department been tasked with treating patients admitted to the inpatient ward for COVID-19?

- No

- Yes, but only a few

- Yes, all members

\section{Multidisciplinary Teams and Lung Cancer}

10. Was it possible to maintain the routine work of the multidisciplinary tumour board at your hospital?

- No, they've been suspended

- Yes, but with social distancing and minimizing the number of assistants

- Yes, using e-platform

- Yes, but less frequently

11. In patients with early-stage lung cancer, did the pandemic alter the treatment decisions made by the MTB?

- Not at all

- $\quad$ Scheduled for surgery only

- A bigger number are scheduled for radiotherapy (SBRT)

- $\quad$ Patients transferred to other centers for surgery

12. In patients with locally-advanced lung cancer, did the pandemic alter the treatment decisions made by the MTB?

- Not at all

- More patients have been scheduled to surgery

- More patients have been scheduled for systemic therapy

- Patients transferred to other centers for surgery

13. What is your opinion regarding the changes in the management of patients with lung cancer?

- I agree on how it is being acted

- I think other centers should be designated as non-COVID-19 in order to maintain surgical activity

- I think other areas in the same hospital should be designated as non-COVID-19 in order to maintain surgical activity

14. Since the start of the pandemic, what is the average waiting time for surgery in your cancer patients?

- $\quad<1$ month

- $1-2$ months

- 2-3 months

- $\quad>3$ months 
Table 1. Cont.

\section{Screening}

15. Did you preoperatively test for SARS-CoV-2 in patients scheduled to undergo thoracic surgery?

- $\quad$ Every patient is screened. This test is necessary before going into the OR

- None

- Only symptomatic patients

16. In patients admitted for thoracic surgery, what was the indication to perform a preoperative diagnostic test for SARS-CoV-2?

- None

- $\quad$ Every patient

- Only symptomatic patients

17. What type of diagnostic tests for SARS-CoV-2 are routinely performed at your center?

- Nasopharyngeal swab

- Sputum

- Blood test for antibodies

- Bronchoalveolar lavage

- Chest X-Ray

- Chest CT

18. In your opinion, when should screening for SARS-CoV-2 infection be performed?

- $\quad$ Every patient before surgery

- $\quad$ Every patient after surgery

- Only when symptomatic

19. In patients with a positive preoperative SARS-CoV-2 test result, does this influence surgical planning in any way?

- $\quad$ The patient goes through surgery. Health worker wear necessary protection.

- $\quad$ Surgery will be postponed at least 14 days.

- $\quad$ Surgery will be postponed only if patient is symptomatic.

- Yes, surgery is suspended and referred for alternative treatment.

\section{Preoperative Workup}

20. How has the COVID-19 pandemic influenced the preoperative workup?

- Not at all, all investigations are available in a normal time frame

- Only pet-CT investigations are delayed, or unavailable

- $\quad$ Only endobronchial investigations (bronchoscopy, EBUS) are delayed or unavailable

- $\quad$ CT guided biopsy is not routinely available

- $\quad$ Pneumology consultation (lung nodule study) is delayed or unavailable

21. Which of the following preoperative lung function tests (pulmonary function testing, pulmonary diffusion test, ... ) are not available due to the COVID-19 pandemic?

- All investigations are available as normal

- $\quad$ Spirometry with or without arterial blood analyses and DLCO

- $\quad$ Cardiopulmonary exercise test (CPET)

- V/Q scan

22. What is your opinion with regard to changes in the preoperative workup?

- I agree according to the situation

- We should minimize the number of tests

- We should do more tests

23. How has the pandemic affected consultations in thoracic surgery?

- Nothing, everything continues with the same operation

- $\quad$ Everything has been suspended

- $\quad$ The face-to-face visits of new patients and the first post-operative visits are maintained. The rest is done electronically.

- All consultations are made electronically 
Table 1. Cont.

\section{Surgery}

24. How has the pandemic affected surgical activity in your department?

- $\quad$ Everything remains the same

- Everything has been suspended

- Benign pathologies have been discontinued. The rest have not been altered

- Exclusively operated on tumors with priority class I (American College of Surgeons classification)

https:/ / www.facs.org/covid-19/clinical-guidance/elective-case/thoracic-cancer (accessed on 20 February 2021)

- $\quad$ Only emergencies are operated

25. What is your opinion with regard to these changes in surgical interventions?

- I agree

- I agree but I think we should further reduce surgical activity

- I do not agree. Under these conditions, the care of my patients is suboptimal

- I do not agree. An effort should be made to operate on more patients

- Other

26. Has the postoperative length of stay in the ICU/recovery unit been affected by the pandemic?

- No

- Yes. The stay in reanimation unit has been reduced

- Yes. Patients do not stay in reanimation unit and go directly to the ward

27. Were any of the patients admitted to your department (regardless of surgical status) diagnosed with SARS-CoV-2?

- None

- One case

- $\quad<5$ cases

- $\quad>5$ cases

28. What recommendations did you use for surgical planning in your department?

- $\quad$ The classification proposed by the American College of Surgeons

- None in particular. We act according to availability and common sense

- There is nothing to prioritize since everything has been suspended

- $\quad$ The links on the SECT website have been very useful for decision-making

- $\quad$ Other (specify)

29. If surgical activity has continued at your hospital, have you observed any increase in morbidity and/or mortality?

- $\quad$ No

- $\quad$ Yes, possibly attributed to the shortage of material and human resources

- Yes, but I don't think it is related to the COVID-19 epidemic

- Yes, the morbidity and mortality has increased because of COVID-19

- $\quad$ Other (specify)

30. What types of surgical interventions (if any) have been performed in your department on COVID-19 patients?

- Thoracic drains due to pneumothorax

- Thoracic drains due to pleural effusion

- Tracheostomies

- $\quad$ Other (specify)

31. Has the pandemic affected the management of pleural fluid drainage?

- No, is still the same

- Yes, we have increased home discharges with drainage

- Yes, we do not discharge anyone with drainage

32. Have the criteria for the drainage tube removal at your department been modified?

- $\quad$ No

- Yes, we remove drains sooner

- Yes, we remove drains later

33. If the patient is discharged to home with a chest tube, what type of system do you use?

- $\quad$ Digital device

- Collection bag

- Dry drain

- Other (specify) 
Table 1. Cont.

\section{Personal Protection}

34. What type of protections are used in surgical procedures for patients who have not been tested for SARS-CoV-2 or whose status is unknown?

- $\quad$ Standard measures

- $\quad$ FFP2/FFP3 and face shields for all attendees

- $\quad$ FFP2/FFP3 and face shields for surgeons. Complete PPE for the anesthesiologist

- Only when it comes to an airway opening procedure, complete PPE for everyone

- $\quad$ Everyone with full PPE

35. What types of protections are used in surgical procedures for patients who test negative for SARS-CoV-2?

- $\quad$ Standard measures

- $\quad$ FFP2/FFP3 and face shields for all attendees

- $\quad$ FFP2/FFP3 and glasses for surgeons. Complete PPE for the anesthetist

- Only when it comes to an airway opening procedure, complete PPE for everyone

- $\quad$ Everyone with full PPE

36. What types of protections are used in surgical procedures performed in patients who test positive for SARS-CoV-2?

- Standard measures

- $\quad$ FFP2/FFP3 and glasses for all attendees

- $\quad$ FFP2/FFP3 and glasses for surgeons. Complete PPE for the anesthetist

- $\quad$ Only when it comes to an airway opening procedure, complete PPE for everyone

- $\quad$ Everyone with full PPE

37. Among the department staff, what percentage of members have tested positive for SARS-CoV-2?

- None

- $<25 \%$

- $25-75 \%$

- $>75 \%$

\section{Teaching and Research}

38. What is your opinion with regard to the quality of the studies published to date?

- Low quality, written too fast

- $\quad$ Moderate scientific quality

- High scientific quality, taking into account the situation

39. How many articles about SARS-CoV-2 and/or COVID-19 infection have you read?

- None

- $<5$

- $5-10$

- $\quad>10$

40. If you are a resident, how has the pandemic has affected your training?

- It has affected me positively

- It has not affected me

- It has affected me negatively

\section{Statistical Analysis}

A descriptive analysis of the data obtained from the survey was performed. All results are given as absolute numbers and percentages.

\section{Results}

A total of 471 SECT members were surveyed and 94 completed the survey, for an overall response rate (RR) of $19.2 \%$.

\subsection{Sociodemographic and Hospital-Related Data}

Sociodemographic and hospital-related data are shown in Table 2 and Figure 1. 
Table 2. Survey results: Sociodemographic and hospital-related data.

\begin{tabular}{|c|c|c|}
\hline Question & $\mathbf{N}$ & $\%$ \\
\hline \multicolumn{3}{|l|}{ What country do you work in? } \\
\hline Spain & 90 & 96.8 \\
\hline \multirow[t]{2}{*}{ Other } & 3 & 3.2 \\
\hline & Total 9 & \\
\hline \multicolumn{3}{|l|}{ What type of institution do you work at? } \\
\hline University Public Hospital & 88 & 89.1 \\
\hline Non-University Public Hospital & 1 & 2.1 \\
\hline Private Hospital & 7 & 8.1 \\
\hline \multirow[t]{2}{*}{ University Private Hospital } & 3 & 4.1 \\
\hline & Total 9 & \\
\hline \multicolumn{3}{|l|}{ How many beds does your hospital have? } \\
\hline$\leq 100$ & 3 & 3.2 \\
\hline 100 to 400 & 12 & 12.8 \\
\hline 400 to 800 & 36 & 38.3 \\
\hline \multirow[t]{2}{*}{$>800$} & 43 & 45.7 \\
\hline & Total 9 & \\
\hline \multicolumn{3}{|l|}{ Did your hospital have to increase the number of beds? } \\
\hline No & 18 & 19.1 \\
\hline Yes, but only for ICU beds & 34 & 36.2 \\
\hline Yes & 23 & 24.5 \\
\hline Yes, by adding beds at other facilities (gym, other centers, ... ) & 19 & 20.2 \\
\hline & Total 9 & \\
\hline \multicolumn{3}{|c|}{$\begin{array}{c}\text { Was it necessary to adapt other spaces in the hospital to treat COVID-19 patients (e.g., gymnasium, } \\
\text { library, cafeteria, field hospital, etc.)? }\end{array}$} \\
\hline Yes & 56 & 59.6 \\
\hline \multirow[t]{2}{*}{ No } & 38 & 40.4 \\
\hline & Total 9 & \\
\hline \multicolumn{3}{|l|}{ Did you refer patients from your center to hotels or other facilities? } \\
\hline Yes & 44 & 46.8 \\
\hline \multirow[t]{2}{*}{ No } & 49 & 52.1 \\
\hline & Total 9 & \\
\hline \multicolumn{3}{|l|}{ To what extent was the normal functioning of your hospital affected? } \\
\hline Not at all & 1 & 1.1 \\
\hline Slightly & 6 & 6.4 \\
\hline Moderately & 16 & 17.0 \\
\hline Severely & 47 & 50.0 \\
\hline \multirow[t]{2}{*}{ Extremely } & 24 & 25.5 \\
\hline & Total 9 & \\
\hline \multicolumn{3}{|l|}{ Have healthcare staff been tested for SARS-CoV-2? } \\
\hline No & 4 & 4.3 \\
\hline Yes, everyone & 19 & 20.2 \\
\hline Yes, but only after developing symptoms & 18 & 19.1 \\
\hline Yes, only after exposure to a COVID-19 patient & 3 & 3.2 \\
\hline Yes, after presenting symptoms or exposure to COVID-19 patient & 22 & 23.4 \\
\hline \multirow[t]{2}{*}{ The criteria are not well defined } & 31 & 33.0 \\
\hline & Total 9 & \\
\hline \multicolumn{3}{|c|}{$\begin{array}{l}\text { Has any member of the department been tasked with treating patients admitted to the inpatient } \\
\text { ward for COVID-19? }\end{array}$} \\
\hline None & 56 & 59.6 \\
\hline Only a few members of the department & 23 & 24.4 \\
\hline \multirow[t]{2}{*}{ All members } & 15 & 16.0 \\
\hline & Total 9 & \\
\hline
\end{tabular}




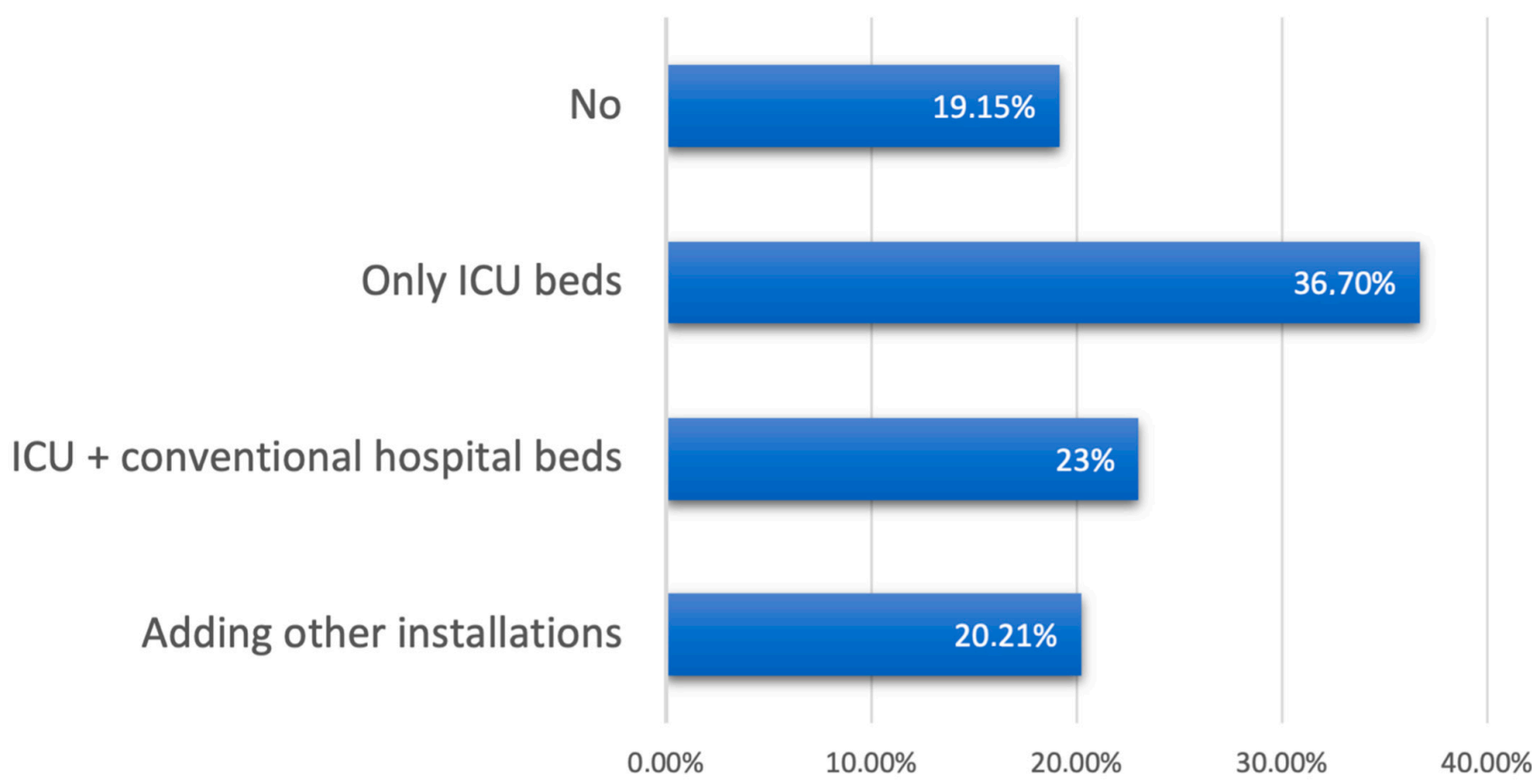

Figure 1. Did your hospital have to increase the number of beds? How?

\subsection{Multidisciplinary Teams and Cancer}

Question 10. Was it possible to maintain the routine work of the multidisciplinary tumour board at your hospital? (RR: 92/94; 97.8\%).

In most of the participating centers, MTB meetings continued to be held during the pandemic. In $44.6 \%$ of centers $(n=41)$, these meetings were held in person but with a reduced number of attendees with distancing to avoid close contact. In $35.9 \%$ of centers $(n=33)$, the meetings were held through electronic platforms. By contrast, $18.5 \%$ of centers $(n=17)$ completely cancelled all MTB meetings (Figure 2A).
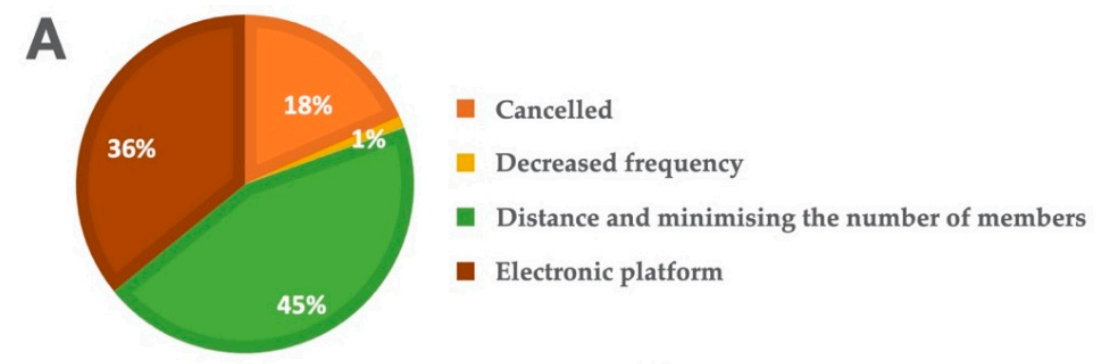

B

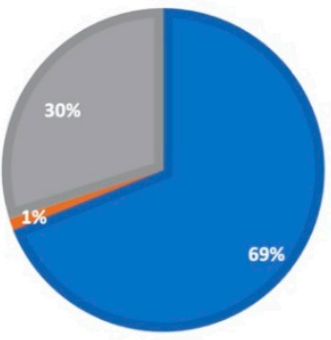

C

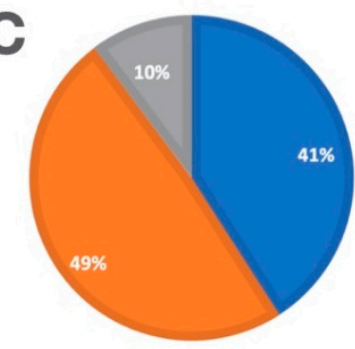

- Not modified

- Not modified

- More patients referred to surgery

- More patients referred to surgery

More patients referred to chemotherapy

- More patients referred to chemotherapy

Figure 2. Multidisciplinary tumour boards. (A) Was it possible to maintain the routine work of the multidisciplinary tumour board at your hospital? (B) In patients with early-stage lung cancer, did the pandemic alter the treatment decisions made by the MTB. (C) In patients with locally-advanced lung cancer, did the pandemic alter the treatment decisions made by the MTB? 
Question 11. In patients with early-stage lung cancer, did the pandemic alter the treatment decisions made by the MTB? (RR: 91/94; 96.8\%).

Nearly $40 \%$ of respondents $(n=36,39.6 \%)$ reported that the pandemic did not influence the management of patients with early-stage lung cancer. However, $47.3 \%(n=43)$ referred more patients to surgery while $9.9 \%(n=9)$ referred more patients to radiation therapy (Figure 2B).

Question 12. In patients with locally-advanced lung cancer, did the pandemic alter the treatment decisions made by the MTB? (RR: 90/94; 95.7\%).

Most centers did not modify the management of patients with locally-advanced disease $(n=60,66.7 \%)$. However, in $28.9 \%$ of centers $(n=26)$, more patients were prescribed chemotherapy. One center $(1.1 \%)$ reported that surgery was indicated in more patients (Figure 2C).

Question 13. What is your opinion regarding the changes in the management of patients with lung cancer? (RR: 92/94; 97.8\%).

Slightly more than half of respondents $(53.3 \% ; n=49)$ agreed with these modifications. By contrast, $43.5 \%(n=40)$ believed that patients should have been referred to COVID-19free centers for treatment.

Question 14. Since the start of the pandemic, what is the average waiting time for surgery in your cancer patients? (RR: 93/94; 98.9\%).

Twenty-nine centers $(31.2 \%)$ reported that waiting time for surgery was less than one month. However, in most centers $(n=47,50.5 \%)$ waiting times ranged from 1 to 2 months. In the remaining centers $(n=16,172 \%)$, the waiting time was $2-3$ months.

\subsection{Patient Screening}

Table 3 summarises the findings regarding patient screening.

Table 3. Survey results on "COVID-19 patient screening" section.

\begin{tabular}{|c|c|c|}
\hline Question & $\mathbf{N}$ & $\%$ \\
\hline \multicolumn{3}{|c|}{ Did you preoperatively test for SARS-CoV-2 in patients scheduled to undergo thoracic surgery? } \\
\hline Every patient is screened. & 91 & 96.8 \\
\hline None & 2 & 2.1 \\
\hline \multirow[t]{2}{*}{ Only if symptomatic } & 1 & 1.1 \\
\hline & Total 94 & \\
\hline \multicolumn{3}{|c|}{$\begin{array}{l}\text { In patients admitted for thoracic surgery, what was the indication to perform a preoperative } \\
\text { diagnostic test for SARS-CoV-2? }\end{array}$} \\
\hline All patients & 39 & 41.9 \\
\hline None & 3 & 3.2 \\
\hline \multirow[t]{2}{*}{ Only if symptomatic } & 51 & 54.8 \\
\hline & Total 93 & \\
\hline \multicolumn{3}{|c|}{ What type of diagnostic tests for SARS-CoV-2 are routinely performed at your centre? } \\
\hline Nasopharyngeal swab & 92 & 98.9 \\
\hline Sputum & 0 & 0.0 \\
\hline Serology & 17 & 18.3 \\
\hline Bronchoalveolar lavage & 0 & 0.0 \\
\hline Chest X-ray & 23 & 24.7 \\
\hline \multirow[t]{2}{*}{ Thorax CT } & 14 & 15.1 \\
\hline & Toal 93 & \\
\hline
\end{tabular}


Table 3. Cont.

\begin{tabular}{|c|c|c|}
\hline Question & $\mathbf{N}$ & $\%$ \\
\hline \multicolumn{3}{|l|}{ In your opinion, when should screening for SARS-CoV-2 infection be performed? } \\
\hline In all patients before surgery & 92 & 98.9 \\
\hline In all patients after surgery & 1 & 1.1 \\
\hline Only in symptomatic cases & 0 & 0.0 \\
\hline & Total 93 & \\
\hline \multicolumn{3}{|c|}{$\begin{array}{l}\text { In patients with a positive preoperative SARS-CoV-2 test result, does this influence surgical planning } \\
\text { in any way? }\end{array}$} \\
\hline No modification of surgical plan & 4 & 4.0 \\
\hline Postpone surgery $>14$ days & 82 & 88.0 \\
\hline Postpone only if symptoms & 5 & 5.0 \\
\hline \multirow[t]{2}{*}{ Surgery is suspended and patient is referred for alternative treatment } & 2 & 2.0 \\
\hline & Total 93 & \\
\hline
\end{tabular}

\subsection{Preoperative Workup}

Question 20. How has the COVID-19 pandemic influenced the preoperative workup? (RR: 92/94; $97.8 \%$ )

A total of 37 respondents (40.2\%) responded that preoperative workup was unchanged from the pre-pandemic period. Among the centers that reported changes, the preoperative tests that were most affected (i.e., delays and/or omissions) were: bronchoscopy (56.5\% of centers), CT-guided biopsy (42.4\%), and referral to the pneumology department for assessment of lung nodules (29.4\%).

Question 21. Which of the following preoperative lung function tests (pulmonary function test, pulmonary diffusion test ... ) are not available due to the COVID-19 pandemic? (RR: 94/94; 100\%)

Forty participants (42.5\%) responded that all tests remained the same. Fifty respondents $(53.2 \%)$ indicated that spirometry and pulmonary diffusion tests were unavailable or with reduced availability, while $36(38.3 \%)$ reported a delay or unavailability of positronemission tomography (PET) imaging. Sixteen (17\%) respondents indicated that the pandemic negatively impacted the availability of ventilation-perfusion lung scintigraphy.

Question 22. What is your opinion with regard to changes in the preoperative workup? (RR: 94/94; 100\%)

Most participants $(n=81,86.2 \%)$ agreed with the preoperative tests that were performed. Only one (1.1\%) thought that fewer tests should be carried out while $12(12.8 \%)$ believed that more tests should be performed.

Question 23. How has the pandemic affected consultations in thoracic surgery? (RR: 94/94; 100\%)

Only one respondent $(1.1 \%)$ indicated that the center maintained the same activity level. Three centers $(3.2 \%)$ cancelled all consultations. In most centers $(n=76,80.8 \%)$ the initial consultation and the first postoperative consultation were maintained, while the remaining consultations were performed online. Finally, 14 centers (14.9\%) reported that all consultations were performed through electronic platforms.

\subsection{Surgery}

Question 24. How has the pandemic affected surgical activity in your department? (RR: 94/94; 100\%)

Four respondents $(4.3 \%)$ reported that the activity level was unchanged while $3(3.2 \%)$ cancelled all surgical interventions. A total of 37 respondents (39.4\%) suspended surgical treatment for benign tumours, and 39 (41.5\%) only performed surgery in cancer patients. Eleven centers $(11.7 \%)$ performed only emergency surgeries. 
Question 25. What is your opinion with regard to these changes in surgical interventions? (RR: 94/94; 100\%)

Most participants $(n=59,62.8 \%$ ) agreed with the changes made and two respondents $(2.1 \%)$ believed that this activity should be further reduced. By contrast, 17 respondents $(18.1 \%)$ disagreed with the changes because this implied suboptimal patient care. Sixteen respondents $(17 \%)$ believed that more surgical interventions should be performed.

Question 26. Has the postoperative length of stay in the ICU/recovery unit been affected by the pandemic? (RR: 93/94; 98.9\%)

Most respondents $(n=64,68.8 \%)$ reported that the length of stay remained unchanged. However, 19 (20.4\%) indicated that the stays were shorter while $10(10.8 \%)$ cancelled postoperative stays.

Question 27. Were any of the patients admitted to your department (regardless of surgical status) diagnosed with SARS-CoV-2? (RR: 93/94; 98.9\%)

Most of the participants, 52 (55.9\%) reported having no infected patients in their department. Ten centers $(10.7 \%)$ reported one positive case, 27 centers $(29 \%)$ reported $<5$, and four centers $(4.3 \%)>5$ cases.

Question 28. What recommendations did you use for surgical planning in your department? (RR: 93/94; 98.9\%)

Thirty-three respondents (35.5\%) based surgical planning on the recommendations of the American College of Surgeons (ACS). More than half of respondents $(n=47,50.5 \%)$ based surgical planning on common sense. Eight centers halted all activity and thus patient prioritisation was not necessary. Five centers (5.4\%) based surgical planning on links published on the SECT website (links to studies and international guidelines).

Question 29. If surgical activity has continued at your hospital, have you observed any increase in morbidity and/or mortality? (RR: 91/94 96.8\%)

Most respondents $(n=65,75.6 \%)$ reported that morbidity and mortality rates were unchanged. However, five respondents $(5.8 \%)$ reported a higher morbidity and mortality rates due to the reduced availability of both material and human resources. Five respondents $(5.8 \%)$ reported an increase in morbidity unrelated to the pandemic while five other centres (5.8\%) reported an increase in morbidity associated with COVID-19.

Question 30. What types of surgical interventions (if any) have been performed in your department on COVID-19 patients? (multiple choice response) (RR: 90/94; 95.7\%)

Seven respondents $(7.8 \%)$ indicated that no surgical procedures were performed in COVID-19 patients at their center. However, 61 centers inserted chest tubes for pneumothorax and 56 for pleural effusion. Twenty-four centers performed tracheostomies. In ten centers, chest tubes were inserted to treat empyema $(n=5)$ or hemothorax $(n=5)$. Three centers performed surgical interventions for subcutaneous emphysema. Three centers performed bronchoscopies. Three centers reported performing surgery for tracheostomyrelated complications and five for other causes.

Question 31. Has the pandemic affected the management of pleural fluid drainage? (RR: 94/94; 100\%)

In 69 centers $(73.4 \%)$, the management of pleural fluids was unchanged. However, 20 respondents $(21.3 \%)$ reported that a higher proportion of patients were discharged to home with the drainage catheter still in place. Five centers $(5.3 \%)$ did not discharge patients to home with the catheter in place.

Question 32. Have the criteria for the drainage tube removal at your department been modified? (RR: 94/94; 100\%) 
Most respondents (89.4\%) maintain the same criteria for chest tube removal. However, $6.4 \%$ and $4.3 \%$, respectively, reported removing the chest tubes either earlier or later than usual.

Question 33. If the patient is discharged to home with a chest tube, what type of system do you use? (RR: 86/94 91.5\%)

Most centers $(n=25,29.1 \%)$ used a digital system, followed by dry drains $(n=34$, $35.4 \%)$, Heimlich valves $(n=14,16.3 \%)$, and collection bags $(n=13,15.1 \%)$.

\subsection{Personal Protection}

Table 4 summarises the findings regarding personal protection.

\subsection{Teaching and Research}

The survey results for teaching and research are shown in Table 5.

Table 4. Survey results: Personal protection.

\begin{tabular}{|c|c|c|}
\hline Question & $\mathbf{N}$ & $\%$ \\
\hline \multicolumn{3}{|l|}{ What type of protections are used in surgical procedures for patients who have not been tested for } \\
\hline Standard measures & 15 & 16.3 \\
\hline FFP2/FFP3 and face shields for the entire surgical team & 43 & 46.7 \\
\hline FFP2/FFP3 and face shields for surgeons. Complete PPE for the anesthesiologist & 16 & 17.4 \\
\hline Only for airway opening procedures. Complete PPE for everyone. & 10 & 10.9 \\
\hline \multirow[t]{2}{*}{ Everyone with full PPE } & 22 & 23.9 \\
\hline & Total 92 & \\
\hline \multicolumn{3}{|l|}{$\begin{array}{l}\text { What types of protections are used in surgical procedures for patients who test negative for } \\
\text { SARS-CoV-2? }\end{array}$} \\
\hline Standard measures & 44 & 46.8 \\
\hline FFP2/FFP3 and face shields for all attendees & 42 & 44.7 \\
\hline FFP2/FFP3 and face shields for surgeons. Complete PPE for the anesthesiologist & 9 & 9.6 \\
\hline Only for airway opening procedures. Complete PPE for everyone. & 5 & 5.3 \\
\hline \multirow[t]{2}{*}{ Everyone with full PPE } & 2 & 2.1 \\
\hline & Total 94 & \\
\hline \multicolumn{3}{|l|}{$\begin{array}{l}\text { What types of protections are used in surgical procedures performed in patients who test positive for } \\
\text { SARS-CoV-2? }\end{array}$} \\
\hline Standard measures & 0 & 0.0 \\
\hline FFP2/FFP3 and face shields for the entire surgical team & 6 & 6.5 \\
\hline FFP2/FFP3 and face shields for surgeons. Complete PPE for the anesthesiologist & 12 & 13.0 \\
\hline Only for airway opening procedures. Complete PPE for everyone. & 3 & 3.3 \\
\hline \multirow[t]{2}{*}{ Everyone with full PPE } & 73 & 79.3 \\
\hline & Total 92 & \\
\hline \multicolumn{3}{|l|}{ Among the department staff, what percentage of members have tested positive for SARS-CoV-2? } \\
\hline None & 45 & 48 \\
\hline$<25 \%$ & 17 & 18 \\
\hline $25-50 \%$ & 21 & 22 \\
\hline $50-75 \%$ & 9 & 10 \\
\hline \multirow[t]{2}{*}{$>75 \%$} & 2 & 2 \\
\hline & Total 94 & \\
\hline
\end{tabular}

PPE: Personal Protective Equipment. 
Table 5. Survey results: Teaching and research.

\begin{tabular}{|c|c|c|}
\hline Question & $\mathbf{N}$ & $\%$ \\
\hline \multicolumn{3}{|c|}{$\begin{array}{l}\text { What is your opinion with regard to the quality of the studies published } \\
\text { to date? }\end{array}$} \\
\hline Low quality, written too fast & 20 & 21.5 \\
\hline Moderate scientific quality & 51 & 54.8 \\
\hline High scientific quality given the situation & 22 & 23.7 \\
\hline & Total 93 & \\
\hline \multicolumn{3}{|c|}{$\begin{array}{l}\text { How many articles about SARS-CoV-2 and/or COVID-19 infection have } \\
\text { you read? }\end{array}$} \\
\hline None & 1 & 1.1 \\
\hline$<5$ & 13 & 13.8 \\
\hline $5-10$ & 30 & 31.9 \\
\hline$>10$ & 50 & 53.2 \\
\hline & Total 94 & \\
\hline \multicolumn{3}{|c|}{ If you are a resident, how has the pandemic has affected your training? } \\
\hline It has affected me positively & 3 & 12.5 \\
\hline It has not affected me & 9 & 37.5 \\
\hline \multirow[t]{2}{*}{ It has affected me negatively } & 12 & 50.0 \\
\hline & Total 24 & \\
\hline
\end{tabular}

\section{Discussion}

The findings of this survey of thoracic surgeons reveals the impact of the COVID-19 pandemic on the surgical treatment of patients with lung cancer in Spain. Our data show that the pandemic had a major impact on clinical activity in thoracic surgery departments in Spain, one of the countries most affected by the first wave of COVID-19. The high incidence of COVID-19 in our country substantially increased demand for health care services, overwhelming the capacity of many hospitals within the Spanish National Health System, with a direct negative impact on mortality rates. Nearly half $(47.3 \%)$ of the survey respondents reported that their hospital had to add ICU and hospital beds, and many were also obliged to refer patients to external facilities (field hospitals, hotels, fairgrounds), which were made available to meet the health care demand. This near collapse of the health care system was confirmed by the survey respondents, three-quarters of whom considered the situation in their hospital as either "very serious" (50\% of respondents) or "critical" (25.5\%). In this context, the Spanish Association of Surgery (AEC) developed a classification system with five alert levels to adjust surgical recommendations according to variations in the epidemiological status of the country over time [20].

The overwhelming demand for hospital care during the pandemic had a direct negative impact on the management of patients with a confirmed or suspected diagnosis of lung cancer. The results of our survey clearly reflect the impact of the pandemic on the diagnostic/therapeutic process in these patients. For example, most respondents $(60 \%)$ reported delaying (or omitting) many preoperative tests due to the pandemic. In addition, some preoperative studies - such as pulmonary function testing or diagnostic procedures such as bronchoscopy or CT-guided transthoracic biopsy-were deferred or directly obviated in certain cases, a strategy that was considered unavoidable due to the epidemiological situation at that time.

Several proposals were made to mitigate the effects of the pandemic. In May 2020, the Society for Advanced Bronchoscopy published recommendations on performing bronchoscopies and airway management in patients during the epidemic, with an emphasis on stratifying outpatient bronchoscopies according to the patient's clinical diagnosis and the urgency of the procedure. In patients with early-stage, resectable lung nodules or masses, those recommendations called for performing outpatient bronchoscopy within two weeks [21]. By contrast, in a survey conducted by the Asian Society for Cardiovascular and Thoracic Surgery, nearly three-fourths (73\%) of respondents recommended omitting perioperative bronchoscopy, endobronchial ultrasound (EBUS), and electromag- 
netic navigation-guided bronchoscopy due to the elevated risk of infection during the procedure [22]. Some international clinical guidelines recommended performing only critical respiratory function tests (e.g., pulmonary function tests prior to lung resection surgery) while avoiding all non-essential tests due to the risk of exposure to the virus [23].

The pandemic also had a direct effect on meetings of the MTB, which in turn impacted decision-making in patients with lung cancer. Interestingly, despite the high hospital demand and case overload, only $3 \%$ of participating centers in this survey reduced the frequency of MTB meetings. This finding is worth highlighting given that the outsized impact of the virus in Spain compared to many other European countries. In fact, a survey carried out by the European Society of Thoracic Surgery (ESTS) found that $20 \%$ of MTB meetings were cancelled in member countries versus only 3\% in Spain [24]. One of the reasons for the low cancelation rate in Spain could be the rapid uptake of online meetings (one-third of which were held online), indicating a rapid capacity for adaptation or perhaps the existence of previously-established digital logistics plan. In this regard, it would be interesting to determine if the proportion of virtual meetings has increased significantly over time. Several international recommendations emphasise the key role that the MTB plays in ensuring the optimal treatment of patients with thoracic malignancies during the pandemic [25].

Approximately two-thirds of the survey respondents reported that the mean waiting time for surgery in patients with a diagnosis or high suspicion of lung cancer was longer than one month. Some respondents suggested that implementation of special "COVID-free" hospitals could have decreased wait times. These long waiting times, attributable to the exceptional circumstances of the pandemic, led some institutions to consider alternatives to surgery. For example, $10 \%$ of respondents offered stereotactic body radiotherapy (SBRT) to patients with early-stage disease and nearly $30 \%$ of respondents reported a decrease in surgical resections for locally-advanced lung cancer. These changes in standard clinical practice may be at least partially attributable to preliminary data published around the time that this survey was performed suggesting a high rate of postoperative morbidity and mortality in complex COVID-19 patients. In fact, this would explain why surgeons initially avoided or delayed performing high-risk procedures, including neoadjuvant treatments. This reduction in surgical resection rates is slightly higher than the figures reported in the ESTS survey [24]. The absence of clear recommendations on elective and urgent thoracic surgery underscores the crucial role of MTBs in decision-making [17].

Given the increased risk of postoperative complications in COVID-19-positive patients, preoperative screening programs for SARS-Cov-2 were implemented for all patients scheduled for surgery. In fact, nearly all of the respondents indicated that preoperative PCR testing was considered essential in surgical patients. The relative importance of the various clinical and radiological screening tests has been studied [20] and it is clear that preoperative molecular testing should be performed as close as possible to the surgical intervention. However, the criteria used to determine patient eligibility for surgery after a positive test for COVID-19 (symptomatic or not) has not yet been established. Nonetheless, the available data suggests that delaying surgery in asymptomatic patients with a positive PCR test does not appear to be associated with higher postoperative morbidity and mortality rates [26].

The shortage of hospital resources (both material and human) due to the combination of high demand and reduced health care services during the first months of the pandemic made it necessary to prioritise patients on thoracic surgery waiting lists, with cancer patients (particularly those with advanced disease) given first priority. On 4 April 2020, the Thoracic Surgery Outcomes Research Network published consensus recommendations for triaging surgery in patients with thoracic malignancies [16], representing an important effort by the international thoracic surgery community to improve decision-making during the pandemic. This consensus statement classified thoracic malignancies and specific surgical procedures into three independent categories: priority, potentially-deferrable, and deferrable. 
In our survey, $41.5 \%$ of the respondents indicated that, during the pandemic, surgeries were restricted to patients with cancer. More than one-third of these professionals (35.5\%) followed the recommendations of the aforementioned consensus statement [16], with most of the others taking a common-sense approach to decision making. In July 2020, the International Association for the Study of Lung Cancer (IASLC) published detailed guidelines for the management of patients with lung cancer [27]. The Spanish Society of Thoracic Surgery, based on data from various international consensus statements and on the preliminary results of the present survey, published its own recommendations for triaging patients with thoracic neoplasms in December 2020 [17]. Our survey showed that confidence in the published recommendations was high, with close to $80 \%$ considering these recommendations to be of moderate to high scientific quality, despite being based on very early data.

Since a high percentage of thoracic surgeries are performed in cancer patients, most of whom are high-risk, it can be difficult to strike a proper balance between the indication for surgery versus the risk of exposure to SARS-CoV-2, especially given that some published reports suggested that mortality rates in COVID-19-positive patients may be up to 30 times greater than patients without COVID-19, with a similarly elevated risk of pulmonary complications [28]. Nevertheless, in our survey, most respondents (71\%) did not report high morbidity and mortality rates, which is especially remarkable considering that this patient cohort was largely comprised of non-deferrable cancer patients. Furthermore, only $11 \%$ attributed worse outcomes to COVID-19.

The negative impact of deferring surgical resection in patients with lung cancer is well known. Therapeutic delay has been shown to reduce survival rates, even in patients with early-stage disease (starting with stage IA2 adenocarcinoma and stage IB squamous carcinoma) [29]. As the survey was performed during the first phase of the pandemic, we do not have information about how any delays in diagnosis or detection of lung cancer might have affected the disease in terms of size, stage, or eligibility for curative surgery. However, previously published studies have demonstrated that cancer screenings, visits, treatment, and surgery have decreased by up to $70 \%$ [30]. As a result, cancer morbidity and mortality is expected to increase for years to come. Given the need to postpone surgery in these patients during the pandemic, some authors proposed SBRT as an alternative treatment in patients with early-stage lung cancer as a "bridge" until surgery could be performed [31]. However, to date, no clear guidelines have been proposed regarding the indications for these alternative therapeutic strategies - which are associated with worse outcomes-in part because the use of these alternative approaches require an individualised assessment by the MTB. In the context of the current pandemic, some reviews have proposed omitting surgery in resectable, locally-advanced lung cancer; however, in our view as surgeons, this would deprive the patient of the most effective therapeutic strategy [32].

This study has several limitations, including possible biases related to the questionnaire design and wording. In addition, our sample was mainly comprised of thoracic surgeons at Spanish hospitals, which limits the sample size and does not provide a multidisciplinary perspective. Only limited data are available to assess the true impact of the pandemic on surgical activity and outcomes for NSCLC in Spain. The GRAVID study carried out by the Spanish Lung Cancer Group offers a general perspective about patients with lung cancer and COVID-19, but it did not evaluate surgical issues [33]. Nevertheless, the survey provides a global perspective of the situation in thoracic surgery departments during the COVID-19 pandemic, and therefore of the surgical treatment of lung cancer during the initial and most critical time points in one of the countries most affected by the virus. Moreover, it allows us to compare these results with those obtained by other countries to identify similarities and differences. Anyway, to ascertain the true impact of the pandemic on lung cancer mortality rates, multicenter registries (ideally national registries) are needed to accurately determine the impact of the reduction in surgical procedures on the therapeutic options offered to patients. 


\section{Conclusions}

The present survey provides key data on the response of thoracic surgery departments in Spain to the severe-albeit heterogeneous-impact of COVID-19 on the management of lung cancer patients. The findings of this survey underscore the resilience of these professionals during the pandemic, who made every effort to provide these cancer patients with the best treatment possible while minimising the risk of exposure to SARS-CoV-2. The initial lack of common protocols at the onset of the pandemic led to a wide range of strategic responses, with a decision-making supported by the experience of multidisciplinary teams.

Author Contributions: Conceptualization: N.J.M.-H.; Methodology: N.J.M.-H., U.C.S. and A.C.S.; Software: N.J.M.-H., U.C.S. and A.C.S.; Validation, J.L.C.-C.d.l.C.; Formal analysis: U.C.S., A.C.S.; Investigation: N.J.M.-H., U.C.S. and A.C.S., J.L.C.-C.d.l.C., A.O.C., J.R.J.S., S.S.L., Á.C.R., J.L.R.D., S.C., F.C., F.H.T.; Resources: F.C., F.H.T.; Data curation: N.J.M.-H., U.C.S. and A.C.S.; Writing—original draft preparation: N.J.M.-H., U.C.S. and A.C.S., J.L.C.-C.d.I.C., A.O.C., J.R.J.S.; Writing-review and editing: S.S.L., Á.C.R., J.L.R.D., S.C.; Visualization: S.S.L., Á.C.R., J.L.R.D., S.C., F.C., F.H.T.; Supervision: N.J.M.-H.; Project administration: N.J.M.-H. All authors have read and agreed to the published version of the manuscript.

Funding: This research received no external funding.

Institutional Review Board Statement: Given the anonymous character and lack of personal information asked, the present survey did not need an ethical review board approval.

Informed Consent Statement: The survey included in its last screen a click option with this sentence: "I consent that the Spanish Society of Thoracic Surgeons (SECT) to have my data included in the survey, as much as make the use the Society considers, including studies, publications or whatever format the Society considers".

Data Availability Statement: The data presented in this study are available in this article.

Acknowledgments: Members of the Scientific Committee of the Spanish Thoracic Surgery Society are: Néstor J. Martínez-Hernández, Usue Caballero Silva, Alberto Cabañero Sánchez, José Luis Campo-Cañaveral de la Cruz, Andrés Obeso Carillo, José Ramón Jarabo Sarceda, Sebastián Sevilla López, Ángel Cilleruelo Ramos, José Luis Recuero Díaz, and Sergi Call.

Conflicts of Interest: The authors declare no conflict of interest.

\section{References}

1. World Health Organization. Cancer. 2018. Available online: https://www.who.int/news-room/fact-sheets/detail/cancer (accessed on 5 October 2019).

2. Rami-Porta, R.; Bolejack, V.; Crowley, J.; Ball, D.; Kim, J.; Lyons, G.; Rice, T.; Suzuki, K.; Thomas, C.F.; Travis, W.D.; et al. The IASLC Lung Cancer Staging Project: Proposals for the Revisions of the T Descriptors in the Forthcoming Eighth Edition of the TNM Classification for Lung Cancer. J. Thorac. Oncol. 2015, 10, 990-1003. [CrossRef] [PubMed]

3. Howington, J.A.; Blum, M.G.; Chang, A.C.; Balekian, A.A.; Murthy, S.C. Treatment of Stage I and II Non-small Cell Lung Cancer. Chest 2013, 143, e278S-e313S. [CrossRef] [PubMed]

4. Zhu, N.; Zhang, D.; Wang, W.; Li, X.; Yang, B.; Song, J.; Zhao, X.; Huang, B.; Shi, W.; Lu, R.; et al. A Novel Coronavirus from Patients with Pneumonia in China, 2019. N. Engl. J. Med. 2020, 382, 727-733. [CrossRef] [PubMed]

5. World Health Organization. Coronavirus Disease (COVID-2019) Situation Reports. 2020. Available online: https:/ / www.who. int/emergencies/diseases/novel-coronavirus-2019/situation-reports (accessed on 18 February 2021).

6. World Health Organization. WHO Coronavirus Disease (COVID-19) Dashboard. Available online: https:/ / covid19.who.int/ (accessed on 18 February 2021).

7. Data on Country Response Measures to COVID-19. 2021. Available online: https://www.ecdc.europa.eu/en/publications-data/ download-data-response-measures-covid-19 (accessed on 18 February 2021).

8. Government Response to Coronavirus, COVID-19 I USAGov. Available online: https://www.usa.gov/ coronavirus (accessed on 18 February 2021).

9. Massinga Loembé, M.; Tshangela, A.; Salyer, S.J.; Varma, J.K.; Ouma, A.E.O.; Nkengasong, J.N. COVID-19 in Africa: The spread and response. Nat Med. 2020, 26, 999-1003. [CrossRef] [PubMed]

10. Short, K.; Kedzierska, K.; Van De Sandt, C.E. Back to the Future: Lessons Learned From the 1918 Influenza Pandemic. Front. Cell. Infect. Microbiol. 2018, 8, 343. [CrossRef] [PubMed]

11. Brewer, L. Historical notes on lung cancer before and after Graham's successful pneumonectomy in 1933. Am. J. Surg. 1982, 143, 650-659. [CrossRef] 
12. Kontis, V.; Bennett, J.E.; Rashid, T.; Parks, R.M.; Pearson-Stuttard, J.; Guillot, M.; Asaria, P.; Zhou, B.; Battaglini, M.; Corsetti, G.; et al. Magnitude, demographics and dynamics of the effect of the first wave of the COVID-19 pandemic on all-cause mortality in 21 industrialized countries. Nat. Med. 2020, 26, 1919-1928. [CrossRef]

13. Condes, E.; Arribas, J.R. Impact of COVID-19 on Madrid hospital system. Enferm. Infecc. Microbiol. Clin. 2020, 39, 256-257. [CrossRef]

14. Hernández-Tejedor, A.; Sánchez, A.J.M.; Barrientos, A.S.; Varela, I.P. The challenge of an intensive care unit in a fairground. Med. Intensiva 2020, 44, 521-522. [CrossRef]

15. Al-Jabir, A.; Kerwan, A.; Nicola, M.; Alsafi, Z.; Khan, M.; Sohrabi, C.; O’Neill, N.; Iosifidis, C.; Griffin, M.; Mathew, G.; et al. Impact of the Coronavirus (COVID-19) pandemic on surgical practice-Part 1. Int. J. Surg. 2020, 79, 168-179. [CrossRef] [PubMed]

16. Antonoff, M.; Backhus, L.; Boffa, D.J.; Broderick, S.R.; Brown, L.M.; Carrott, P.; Clark, J.M.; Cooke, D.; David, E.; Facktor, M.; et al. COVID-19 Guidance for Triage of Operations for Thoracic Malignancies: A Consensus Statement From Thoracic Surgery Outcomes Research Network. Ann. Thorac. Surg. 2020, 110, 692-696. [CrossRef] [PubMed]

17. Jarabo, J.R.; Martínez, N.; Cabañero, A.; Call, S.; Campo-Cañaveral, J.L.; Cilleruelo, Á.; Gama, M.G.M.; Mendoza, O.E.C.; Gude, V.D.; García, A.P.G. Recommendations for acting in elec-tive and urgent thoracic surgery during SARS-CoV-2 pandemic. Spanish Society of Thoracic Surgery. Cirugia Espanola 2020, 98, 574-581. [CrossRef] [PubMed]

18. Salmerón Jiménez, M.; Hermoso Alarza, F.; Martínez Serna, I.; Marrón Fernández, C.; Meneses Pardo, J.C.; García Salcedo, J.A. Clinical features and outcomes of thoracic surgery patients during the COVID-19 pandemic. Eur. J. Cardiothorac. Surg. 2020, 58, 738-744. [CrossRef] [PubMed]

19. Hoyos Mejía, L.; Romero Román, A.; Gil Barturen, M.; Córdoba Pelaez, M.; del Campo-Cañaveral, M.; de la Cruz, J.L.; Naranjo, J.M. Thoracic surgery during the coronavirus disease 2019 (COVID-19) pandemic in Madrid, Spain: Single-centre report. Eur. J. Cardiothorac. Surg. 2020, 58, 991-996. [CrossRef] [PubMed]

20. Balibrea, J.; Mose Badia, J.; Mose Rubio Pérez, I.; Martín Antona, E.; Álvarez Peña, E.; García Botella, S.; Gallego, M.A.; Pérez, E.M.; Cortijo, S.M.; Miguelan, I.P.; et al. Manejo quirúrgico de pacientes con infección por COVID-19. Recomendaciones de la Asociación Española de Cirujanos. Circ. Esp. 2020, 98, 251-259. [CrossRef]

21. Pritchett, M.A.; Oberg, C.L.; Belanger, A.; De Cardenas, J.; Cheng, G.; Nacheli, G.C.; Franco-Paredes, C.; Singh, J.; Toth, J.; Zgoda, M.; et al. Society for Advanced Bronchoscopy Con-sensus Statement and Guidelines for bronchoscopy and airway management amid the COVID-19 pandemic. J. Thorac. Dis. 2020, 12, 1781-1798. [CrossRef] [PubMed]

22. Jheon, S.; Ahmed, A.D.; Fang, V.W.; Jung, W.; Khan, A.Z.; Lee, J.-M. Thoracic cancer surgery during the COVID-19 pandemic: A consensus statement from the Thoracic Domain of the Asian Society for Cardiovascular and Thoracic Surgery. Asian Car-diovasc. Thorac. Ann. 2020, 28, 322-329. [CrossRef] [PubMed]

23. Crimi, C.; Impellizzeri, P.; Campisi, R.; Nolasco, S.; Spanevello, A.; Crimi, N. Practical considerations for spirometry during the COVID-19 outbreak: Literature review and insights. Pulmonology 2020. [CrossRef] [PubMed]

24. Depypere, L.P.; Daddi, N.; Gooseman, M.R.; Batirel, H.F.; Brunelli, A. The impact of coronavirus disease 2019 on the practice of thoracic oncology surgery: A survey of members of the European Society of Thoracic Surgeons (ESTS). Eur. J. Cardio Thoracic Surg. 2020, 58, 752-762. [CrossRef] [PubMed]

25. Blais, N.; Bouchard, M.; Chinas, M.; Lizotte, H.; Morneau, M.; Spicer, J.; Martel, S. Consensus Statement: Summary of the Quebec Lung Cancer Network Recommendations for Prioritizing Patients with Thoracic Cancers in the Context of the COVID-19 Pandemic. Curr. Oncol. 2020, 27, 313-317. [CrossRef]

26. Baiocchi, G.; Aguiar, S.; Duprat, J.P.; Coimbra, F.J.F.; Makdissi, F.B.; Vartanian, J.G.; Zequi, S.; Gross, J.L.; Nakagawa, S.A.; Yazbek, G.; et al. Early postoperative outcomes among pa-tients with delayed surgeries after preoperative positive test for SARS-CoV-2: A case-control study from a single institu-tion. J. Surg. Oncol. 2021, 123, 823-833. [CrossRef] [PubMed]

27. Dingemans, A.-M.C.; Soo, R.A.; Jazieh, A.R.; Rice, S.J.; Kim, Y.T.; Teo, L.L.; Warren, G.W.; Xiao, S.-Y.; Smit, E.F.; Aerts, J.G.; et al. Treatment Guidance for Patients With Lung Cancer During the Coronavirus 2019 Pandemic. J. Thorac. Oncol. 2020, 15, 1119-1136. [CrossRef] [PubMed]

28. Doglietto, F.; Vezzoli, M.; Gheza, F.; Lussardi, G.L.; Domenicucci, M.; Vecchiarelli, L.; Zanin, L.; Saraceno, G.; Signorini, L.; Panciani, P.P.; et al. Factors Associated With Surgical Mortality and Complications Among Patients With and Without Coronavirus Disease 2019 (COVID-19) in Italy. JAMA Surg. 2020, 155, 691. [CrossRef] [PubMed]

29. Mayne, N.R.; Elser, H.; Darling, A.J.; Raman, V.; Liou, D.; Colson, Y.; D'Amico, T.A.; Yang, C.H. Estimating the Impact of Extended Delay to Surgery for Stage I Non-Small-Cell Lung Cancer on Survival: Lessons for the COVID-19 Pandemic. Ann Surg. 2021, 237, 850-857. [CrossRef] [PubMed]

30. Patt, D.; Gordan, L.; Diaz, M.; Okon, T.; Grady, L.; Harmison, M.; Markward, N.; Sullivan, M.; Peng, J.; Zhou, A. Impact of COVID-19 on Cancer Care: How the Pandemic Is Delaying Cancer Diagnosis and Treatment for American Seniors. JCO Clin. Cancer Inform. 2020, 4, 1059-1071. [CrossRef] [PubMed]

31. Mayne, N.R.; Lin, B.K.; Darling, A.J.; Raman, V.; Patel, D.C.; Liou, D.Z.; D’Amico, T.A.; Yang, C.-F.J. Stereotactic Body Radiotherapy Versus Delayed Surgery for Early-stage Non-small-cell Lung Cancer. Ann. Surg. 2020, 272, 925-929. [CrossRef] [PubMed]

32. Kumar, S.; Chmura, S.; Robinson, C.; Lin, S.H.; Gadgeel, S.M.; Donington, J.; Feliciano, J.; Stinchcombe, T.E.; Werner-Wasik, M.; Edelman, M.J.; et al. Alternative Multidisciplinary Management Op-tions for Locally Advanced NSCLC During the Coronavirus Disease 2019 Global Pandemic. J. Thorac. Oncol. 2020, 15, 1137-1146. [CrossRef] [PubMed]

33. Provencio, M.; Gallego, J.M.M.; Calles, A.; Antoñanzas, M.; Pangua, C.; Rubio, X.M.; Nadal, E.; Castro, R.L.; López-Martín, A.; del Barco, E.; et al. Lung cancer patients with COVID-19 in Spain: GRAVID study. Lung Cancer 2021. [CrossRef] [PubMed] 